\title{
Analisi delle dimensioni culturali e della cortesia linguistica presente nell'articolo La macchina del fango di Umberto Eco
}

\author{
Edoardo Natale \\ Università di Modena \\ edona54@hotmail.com
}

\begin{abstract}
L'articolo intende analizzare il concetto di dimensione culturale proveniente dal quadro della comunicazione interculturale nell'ambito del lavoro teorico di Geert Hofstede e il concetto di cortesia linguistica di Brown e Levinson. L'articolo analizza un articolo di Umberto Eco raccolto dal sito del settimanale Espresso nell'ottobre 2015. Lo scopo dell'articolo è quello di spiegare il meccanismo sottostante della macchina del fango all'interno del sistema mediatico italiano. L'originalità di questo lavoro consiste nell'utilizzare il concetto di dimensioni culturali utilizzato nell'ambito sociologico così come il concetto di faccia della cortesia linguistica applicato a un testo letterario. In conclusione, questo articolo vorrebbe offrire uno strumento interpretativo utile per descrivere alcune questioni ancora valide sulla realtà culturale e politica dell'Italia. L'intento è capire quali dimensioni culturali e faccia come concetto sono coinvolti in questo articolo.
\end{abstract}

Parole chiave: Dimensione culturale. Faccia. Cortesia linguistica. Cultura italiana. Media. Politica.

RESUMO: $O$ artigo busca analisar o conceito de dimensão cultural proveniente do quadro da comunicação intercultural na obra teórica de Geert Hofstede e o conceito de cortesia linguística de Brown e Levinson. O trabalho analisa um artigo de Umberto Eco publicado no site do jornal Espresso, em outubro de 2015. O objetivo do artigo é explicar o mecanismo subjacente da macchina di fango no sistema de mídia italiano. A originalidade deste trabalho consiste em utilizar o conceito de dimensões culturais utilizado na sociologia, bem como o conceito de faccia da cortesia linguística aplicado a um texto literário. Enfim, este artigo pretende oferecer uma ferramenta interpretativa útil para descrever algumas questões ainda válidas sobre a realidade cultural e política da Itália.

Palavras-chave: Dimensão cultural. Faccia. Cortesia linguística. Cultura italiana. Mass media. Política.

ABSTRACT: The article aims to analyze the concept of cultural dimension from the framework of intercultural communication within the theoretical work of Geert Hofstede and the concept of politeness linguistic from Brown and Levinson. The article analyzes a article of Umberto Eco collected from the 
website of Espresso Magazine on October 2015. The aim of the article is to explain the meccanism of this macchina del fango inside the Italian media system. The originality of this work is to use a method of analysis used in sociological arena applied to a literary text. In conclusion, this article would like to offer an interpretive tool useful to describe some issues still valid about the cultural and political reality of Italy. The intent is to understand which "cultural dimensions" and "face" as politeness concept are involved during this article.

Keywords: Cultural dimension. Face. Politeness linguistic. Italian culture. Media. Politics.

\section{Introduzione}

Questo articolo intende utilizzare l'articolo La macchina del fango di Umberto Eco apparso nella rivista dell'Espresso nel mese di Ottobre 2015 come corpus per adoperare gli strumenti di analisi della cortesia linguistica, della pragmatica e della dimensione culturale. Il contenuto di questo articolo è sembrato un osservatorio privilegiato per cogliere alcuni meccanismi presenti nel paese da tanti anni. L'acutezza di sguardo di Umberto Eco restituiscono con chiarezza una maggiore comprensione della scena politico-mediatica italiana degli ultimi 20 anni. Tali osservazioni, accompagnate con gli strumenti della dimensione culturale di Gert Hofstede e il concetto di faccia di Brown e Levinson, trovano una luce ancora più viva e brillante in questa nuova cornice.

\section{Premessa metodologica}

L'articolo intende analizzare il concetto di dimensione culturale proveniente dal quadro di riferimento della comunicazione interculturale con il lavoro del teorico Geert Hofstede.

Il concetto di dimensione culturale viene definito nei lavori di Hofstede come ambito antropologico problematico dove le varie società gestiscono in modo differente questi ambiti antropologici problematici. Queste dimensioni 
culturali vengono identificate con l'elaborazione di sei dimensioni utili: forte o debole indice di distanza sociale dal potere, forte o debole evitamento dell'incertezza, individualismo versus collettivismo, mascolinità versus femminilità, orientamento a lungo termine o breve termine. L'ultima dimensione viene definita da Hofstede di tipo indulgence versus restraint. Per questa dimensione ho optato per il contesto della società italiana per le nozioni di soddisfazione, appagamento e restrizioni, vincoli. Questo concetto di dimensione culturale è da intendersi come una preferenza verso una dimensione piuttosto che un'altra durante il nostro agire quotidiano in termini di valori. Ogni dimensione possiede al suo interno vari elementi di analisi per meglio identificare la dimensione in questione.

\section{A Alto indice di distanza sociale dal potere come dimensione culturale}

La prima dimensione culturale denominata come indice di distanza sociale dal potere prova ad analizzare il tasso di distanza sociale dal potere individuando come parametri operativi da una parte una società a basso indice di distanza sociale dal potere e al suo opposto abbiamo una società con elevato indice di distanza sociale dal potere.

I parametri che caratterizzano una società a basso indice di distanza sociale sono nel quadro di riferimento di Hofstede:

- un utilizzo del potere che dovrebbe essere legittimato ed è soggetto ai criteri di bene o male

- i genitori trattano i figli come pari

- le persone anziane non sono né rispettate né temute

- l'istruzione viene incentrata sullo studente

- la gerarchia significa un'ineguaglianza dei ruoli ed è stabilita per convenienza 
- gli impiegati o subalterni si attendono di essere consultati

- i governi sono di tipo pluralistici e tendono a cambiare in modo pacifico

- la corruzione è rara e gli scandali portano alla fine della carriera politica

- la ridistribuzione delle tasse è piuttosto elevata

- la religione enfatizza l'uguaglianza dei credenti

I parametri che caratterizzano una società ad alto indice di distanza sociale sono:

- il potere costituisce un fatto basico della società ed è antecedente ai criteri di bene o male. La sua legittimità è irrilevante

- i genitori insegnano l'obbedienza ai figli

- gli anziani vengono rispettati e temuti

- l'istruzione è centrata sul ruolo del docente

- la gerarchia equivale a ineguaglianza esistenziale

- i dipendenti si aspettano che gli venga detto quello che devono fare

- i governi sono autocratici e si fondano sulla cooptazione e vengono rimossi tramite drastici cambiamenti o rivoluzioni

- la corruzione è frequente e gli scandali vengono coperti

- la ridistribuzione delle tasse è molto debole

- la religione è costituita da una gerarchia di preti

\section{B La dimensione culturale detta come forte o basso evitamento dell'incertezza}

La seconda dimensione culturale presente nel quadro di riferimento di Hofstede si definisce con il nome di debole o forte senso di evitamento dell'incertezza.

I parametri per analizzare una società a debole evitamento dell'incertezza sono i seguenti: 
Analisi delle dimensioni culturali e della cortesia linguistica presente

nell'articolo La macchina del fango di Umberto Eco

Edoardo Natale

- l'inerente incertezza della vita viene accettata ed ogni giorno viene preso così come viene

- le persone sono tranquilli, poco stress, controllo di sé e bassa ansietà

- tendenza al benessere personale e allo star bene

- tolleranza verso le persone e le idee divergenti, la differenza è curiosa

- a proprio agio con ambiguità e caos

- gli insegnanti possono dire "non lo so"

- cambiare lavoro non è un problema

- non si apprezzano le regole scritte o non scritte

- nella politica, i cittadini sono visti come competenti agli occhi delle autorità

- nella religione, la filosofia sono preponderanti il relativismo e lo spirito empirico

I parametri per analizzare una società a forte evitamento dell'incertezza sono i seguenti:

- l'incertezza della vita viene sentita come una permanente minaccia che va combattuta

- presenza elevata di stress, emozioni, ansietà e nevrotismo

- poca considerazione per il proprio benessere e lo star bene

- intolleranza verso persone ed idee divergenti, la differenza è pericolosa

- forte bisogno di chiarezza e strutture

- gli insegnanti possiedono tutte le risposte

- conservare il lavoro anche se non ti piace

- bisogno emotivo di regole anche se non sempre rispettate

- in politica, i cittadini sono visti come incompetenti dalle autorità 
Analisi delle dimensioni culturali e della cortesia linguistica presente

nell'articolo La macchina del fango di Umberto Eco

Edoardo Natale

- nella religione e nella filosofia si crede nella verità e nelle grande teorie

\section{C Dimensione culturale di tipo individualismo o collettivismo}

Un'altra dimensione riguarda la differenza tra società con dimensioni culturali di tipo collettivismo da quelle di tipo individualismo.

I parametri per analizzare la dimensione di tipo individualismo sono i seguenti:

- tutti sono tenuti a badare a se stessi e ai membri stretti di famiglia

- "Io" di tipo coscienzioso

- diritto alla privacy

- parlare per sé è cosa buona

- gli altri sono considerati come individui

- opinioni personali ricercata: una persona un voto

- la trasgressione delle norme genera sentimenti di colpevolezza

- Io è una parola indispensabile nel nostro linguaggio

- lo scopo dell'istruzione è imparare ad imparare

- i compiti sono più importanti delle relazioni

I parametri per analizzare la dimensione del "collettivismo" sono così elencati:

- le persone aderiscono a famiglie o clan allargati che offrono protezione in cambio di lealtà

- noi di tipo coscienzioso

- enfatizzazione dell'appartenenza

- il mantenimento dell'armonia è prevalente

- gli altri percepiti come membri in-group o out-group 
Analisi delle dimensioni culturali e della cortesia linguistica presente nell'articolo La macchina del fango di Umberto Eco

Edoardo Natale

- opinioni e votazioni sono dettate dall'appartenenza al proprio in-group di riferimento

- trasgredire le regole porta a sentimenti di vergogna

- Io è una parola a sentimenti di vergogna

- lo scopo dell'istruzione è quello di imparare come fare le cose

- le relazioni sono più importanti dei compiti

\section{D Dimensione culturale di tipo femminilità versus mascolinità}

Un'altra dimensione culturale presente nei lavori di Hofstede riguarda la differenza tra società di tipo femminili o di tipo maschili.

I parametri per analizzare la dimensione della femminilità sono:

- differenza di genere bassa in termini sociali ed emozionali

- gli uomini e le donne devono apparire modesti ed intenzionati

- equilibrio tra vita di famiglia e lavoro

- il padre e la madre gestiscono i fatti e le emozioni

- uomini e donne possono piangere ma entrambi devono combattere

- le madri decidono il numero di figli

- molte donne partecipano alla vita politica

- la religione si concentra sugli esseri umani

- la sessualità è un dato di fatto ed un modo di comunicazione

I parametri per analizzare la dimensione della mascolinità sono:

- differenza di genere elevata in termini di ruoli sociali ed emozionali

- gli uomini devono essere assertivi ed ambiziosi mentre le donne potrebbero esserlo

- il lavoro prevale sulla famiglia

- il padre gestisce i fatti e la madre gestisce le emozioni 
Analisi delle dimensioni culturali e della cortesia linguistica presente nell'articolo La macchina del fango di Umberto Eco

Edoardo Natale

- le donne piangono, gli uomini non possono; gli uomini devono lottare mentre le donne non dovrebbero. Il padre decide la taglia della propria famiglia.

- poche donne partecipano alla vita politica

- la religione si concentra su Dio

- attitudine moralistica verso la sessualità; il sesso è visto come un atto performativo

\section{E Dimensione culturale con orientamento temporale a breve termine oppure a lungo termine}

Un'altra dimensione riguarda la differenza tra società ad orientamento temporale di breve termine oppure società caratterizzate per un rapporto con il tempo fondato su di un orientamento di lungo termine.

I parametri per analizzare la dimensione dell'orientamento a breve termine sono:

- i fatti più importanti della vita sono avvenuti nel passato o in questo momento

- la stabilità della persona intesa come sempre uguale a se stessa

- ci sono delle linee universali a proposito di ciò che è bene o male nelle situazioni

- le tradizioni sono sacrosante

- la vita famigliare è guidata da imperativi

- essere orgogliosi del proprio paese

- essere al servizio degli altri è importante

- spendere soldi per la vita sociale e il consumo

- studenti attribuiscono il successo e il fallimento alla fortuna

- bassa crescita per i paesi poveri 
I parametri per analizzare la dimensione dell'orientamento a lungo termine sono:

- i fatti più importanti della vita devono succedere nel futuro

- una persona brava si adatta alle circostanze

- il bene o il male dipende dalle circostanze

- le tradizioni sono modificabili al cambiamento

- la vita famigliare viene guidata da compiti condivisi

- cercare di imparare dagli altri paesi è cosa buona

- il risparmio e la perseveranza sono obiettivi veri

- attitudine al risparmio e soldi disponibili in caso di investimento da compiere

- studenti attribuiscono il successo allo sforzo e il fallimento alla mancanza di sforzo

- crescita elevata per i paesi con un certo livello di prosperità

\section{F Dimensione culturale presente tra società di tipo}

\section{soddisfate/appagate o di tipo vincolate}

L'ultima dimensione viene definita da Hofstede nei suoi lavori come di tipo indulgence versus restraint. Per questa dimensione ho optato per il contesto della società italiana per le nozioni di soddisfaz̨ione, appagamento e restrizioni, vincoli che mi sembrano dei lessemi maggiormente in consonanza con il contesto culturale dove un termine come indulgente per parlare di una dimensione culturale comporta un maggiore grado di difficoltà interpretativo.

In questo lavoro, i parametri per analizzare la dimensione di una società del tipo soddisfate/appagate sono i seguenti:

- molte persone si dichiarano felici 
Analisi delle dimensioni culturali e della cortesia linguistica presente

nell'articolo La macchina del fango di Umberto Eco

Edoardo Natale

- una sensazione di controllo della propria vita

- la libertà di parola sembra un fatto importante

- tendenza a ricordare le emozioni positive

- elevato tasso di nascita nei paesi molto istruiti

- elevato numero di persone obese in paesi ricchi

- tolleranza verso i comportamenti sessuali

- la sicurezza della nazione non rappresenta una priorità importante

I parametri per analizzare la dimensione di una società di tipo vincolata sono i seguenti:

- poche persone si dicono felici

- un sentimento di abbandono nelle persone: le cose non dipendono dal mio volere

- la libertà di parola non è di prima necessità

- poca importanza al tempo libero

- meno propensi a ricordare le emozioni positive

- basso tasso di nascita nei paesi ricchi

- poche persone fanno sport

- poche persone obese nei paesi ricchi

- rispetto delle norme sessualità

- numero elevato di forze di polizia per il numero di abitanti

\section{La visione della cortesia in Brown e Levinson}

La cortesia linguistica intesa da Brown e Levinson (1978, 1987) riconosce un valore importante alla sua formulazione teorica come sostenuto ad esempio in questo passaggio del libro Politeness. Some universals in use: 


\section{Analisi delle dimensioni culturali e della cortesia linguistica presente nell'articolo La macchina del fango di Umberto Eco \\ Edoardo Natale}

$\ldots$ is at heart of Grice's proposals, namely that there is a working assumption by conversationalists of the rational and efficient nature of talk. It is against that assumption that polite ways of talking show up as deviations, requiring rational explanations on the part of the recipient, who finds in considerations of politeness reasons for the speaker's apparent irrationality or inefficiency". (1987, p. 4).

Per Brown e Levinson, una forte motivazione per non parlare in modo diretto, contravvenendo al rispetto delle massime conversazionali di Grice è da ricercare nel mantenimento della "faccia" come immagine pubblica di sé (Goffman, 1967). Brown e Levinson riconoscono che la cortesia non è l'unica fonte di "deviazione" ai principi di cooperazione introdotti da Grice (1975) ma possiamo ritrovarli anche nell'umorismo, il sarcasmo e l'ironia (Brown e Levinson, 1987).

Brown e Levinson sostengono che la cortesia linguistica dovrebbe essere comunicata e che la sua non comunicazione costituisce un messaggio che comporta un'implicatura conversazionale secondo la teoria griceana (1975). Nel modello di Brown e Levinson, la presenza o mancanza di cortesia si spiega alla luce della cornice ideata da Goffman (1967), ripresa da Brown e Levinson (1987), sul concetto di faccia inteso come un costrutto universale elaborato culturalmente per "la propria immagine pubblica, che ogni membro di una società vuole affermare per se stesso" (1987, p. 61). Brown e Levinson (1987) caratterizzano la faccia come qualcosa che si può perdere, mantenere, o rinforzare durante l'interazione e dato il carattere di vulnerabilità sempre presente della faccia, i parlanti saranno impegnati a difendere la loro faccia se dovesse essere minacciata. Dato il pessimismo secondo Kebrat-Orecchioni (1992, 1996) dello scambio conversazionale nell'approccio di Brown e Levinson, il presupposto è che sia generalmente meglio per l'interesse di tutti conservare la faccia dell'altro e di agire in modo tale che gli altri siano coscienti di questo elemento.

L'idea di fondo che governa la teoria della cortesia di Brown e Levinson 
è vedere che alcuni atti sono di per sé minacciosi per la faccia (es: il disaccordo e la protesta) e pertanto necessitano di essere mitigati. (1987, p. 24). Tuttavia, saranno i lavori di Wierzbicka (1991) ha mettere in luce come ogni gruppo linguistico sviluppi dei principi di cortesia dai quali derivano alcune strategie linguistiche che si rifanno alle norme comunicative presenti in un dato gruppo sociale (WIERZBICKA, 1991).

Per Brown e Levinson (1987) è attraverso la conoscenza di queste strategie di cortesia di natura universale che il parlante ha successo nel comunicare il suo messaggio principale dimostrando in questo modo la sua intenzione di essere cortese e allo stesso tempo di non voler perdere la faccia. In Brown e Levinson vengono presentati degli atti che sono intrinsecamente minacciosi per la faccia del parlante, dell'ascoltatore o per entrambi: ad esempio, il disaccordo viene definito come un atto minaccioso per la faccia positiva dell'ascoltatore insieme ad altri atti linguistici come lamentarsi, criticare o parlare di argomenti tabù. Quando si è costretti a produrre questi atti di minaccia per la faccia degli interagenti, il lavoro di face-work deve concentrarsi sulla riduzione della minaccia come affermano Brown e Levinson:

we have claimed that a face-bearing rational agent will tend to utilize the FTA-Minimizing strategies according to a rational assessment of the face risk to participants. He would behave thus by virtue of practical reasoning, the inference of the best means to satisfy stated ends (1987, p. 91).

Per Brown e Levinson compiere un atto in modo diretto, senza compiere atti di mitigazione, è il modo più chiaro e inequivocabile per compiere un atto di minaccia alla faccia. Invece, in questo modello teorico della cortesia, le strategie di riparazioni possono coinvolgere la cortesia positiva (mostrando solidarietà ) oppure la cortesia negativa ( mostrando distanza).

Così come aveva affermato Leech (1983) nel suo modello di cortesia, 
Brown e Levinson riprendono l'idea di varianti che potrebbero colpire un parlante tramite un FTA ( atto di minaccia per la faccia).

Queste varianti sono indipendenti e sensibili al contesto culturale e giocano un ruolo importante nel modello di Brown e Levinson:

1. la distanza sociale (D) tra il parlante e l'ascoltatore: in pratica, si tratta di capire il grado di famigliarità e di solidarietà condivisa.

2. Potere relativo $(\mathrm{P})$ del parlante nei confronti dell'ascoltatore: di fatto stiamo parlando del grado d'imposizione del parlante sull'ascoltatore.

3. Grado d'imposizione $(\mathrm{R})$ in quella cultura, in termini sia di richiesta di bene e di servizi da parte dell'ascoltatore: in altre parole, il diritto di realizzare alcuni atti e il grado di imposizione che l'ascoltatore può accettare.

Il modello teorico pensato da Brown e Levinson (1987) riconduce "il peso" (indicato come W nella formula) dei nostri atti in termini di faccia positiva o negativa durante la realizzazione di un FTA attraverso una formula, in cui $\mathrm{S}$ indica il parlante, $\mathrm{H}$ indica l'ascoltatore $\mathrm{e} \mathrm{R}$ indica il grado d'imposizione da calcolare in questo modo:

$\mathrm{Wx}=\mathrm{D}(\mathrm{S}, \mathrm{H})+\mathrm{P}(\mathrm{H}, \mathrm{S})+\mathrm{R} \mathrm{x}$

In Brown e Levinson (1987) sarà il valore di Wx ha determinare il grado di cortesia necessario per salvare la propria faccia e queste varianti non devono essere viste come delle costanti tra gli individui. Durante l'interazione, i partecipanti vacillano nella loro distanza sociale quando si trovano ad esempio in situazione di lavoro, oppure in una situazione di nervosismo e allo stesso modo il potere relativo cambia quando i ruoli e le responsabilità cambiano. Quindi la scelta di una data forma linguistica va vista come una realizzazione specifica di una strategia di cortesia alla luce della valutazione del contesto della frase. Questo modello esplicativo di Brown e Levinson può 
essere riassunto in questi passaggi (1987, p. 90-91):

- anche se il parlante intende compiere un FTA ( atto minaccioso per la faccia) con la massima efficienza, il parlante deve determinare se si augura di raggiungere il bisogno di faccia dell'ascoltatore tramite la cooperazione dell'ascoltatore oppure con la conservazione della sua faccia.

- Il parlante dovrebbe determinare la minaccia alla faccia di un dato FTA e determinare fino a quale estensione minimizzare la perdita di faccia dovute all'atto di minaccia, considerando i fattori come il bisogno di chiarezza e quello di non sopravvalutare il grado potenziale di perdita della propria faccia.

- Il parlante deve scegliere una strategia che provveda al grado di "salvare la faccia" con quello menzionato sopra (2). La presa in considerazione della cooperazione dell'ascoltatore determina la strategia scelta per realizzare le aspettative richieste per quell'atto da parte dell'ascoltatore.

- Il parlante deve allora scegliere un significato linguistico che lo soddisfi per la conclusione di quella strategia. Ogni strategia abbraccia un'ampia gradualità di cortesia dove al parlante sarà richiesto di considerare la specifica forma linguistica usata per coglierne gli effetti quando viene usata insieme ad altri elementi linguistici.

Un elemento importante è capire che la scelta di una forma linguistica è determinata dalla responsabilità da parte del parlante verso il suo interlocutore durante l'interazione. Nell'ambito degli studi di pragmatica contrastiva appare necessario integrare la formula pensata da Brown e Levinson per definire il come si conferisce faccia all'interno di varie culture.

\section{Analisi della dimensione culturale del concetto di faccia nell'articolo}

\section{La macchina del fango}


La macchina del fango è un articolo di Umberto Eco apparso nella rivista dell'Espresso nel mese di Ottobre 2015. Questo articolo di Umberto Eco sarà utilizzato come corpus per adoperare gli strumenti di analisi della cortesia linguistica, della pragmatica e della dimensione culturale. In questo lavoro Umberto Eco menziona il fatto che il suo articolo è nato dopo la visita di un gruppo di giornalisti spagnoli, in altri termini questo è un modo per conferire faccia positiva alla propria persona in termini di ricerca di riconoscimento. Allo stesso modo si conferisce faccia positiva alla rivista che accoglie il suo articolo creando un diritto di associazione tra le due esperienze editoriali. Il sottotitolo dell'articolo recita; "perché noi italiani siamo maestri nell'arte dell'insinuazione calunniosa". Questo sottotitolo rappresenta, in un primo momento, un modo per conferire faccia positiva ad un'identità collettiva o faccia pubblica di una nazione per mitigare i costi molto elevati dell'essere maestri, quindi con un forte evitamento dell'incertezza come dimensione culturale, nel compiere una certa attività definita "arte" intesa come una competenza nella "insinuazione calunniosa", la quale potrebbe rientrare nella dimensione culturale di "debole evitamento dell'incertezza" da parte di coloro che compiono tale atto linguistico poiché l'insinuazione non possiede elementi di prova ed è resa possibile da una diffusa adesione ad una dimensione culturale fatta di distanza sociale in cui legittimare i propri propositi è percepito come irrilevante. Inoltre non si risponde alla massima di qualità per dirla alla Grice ( 1975) perché si afferma quello che non si può provare facendo delle allusioni accettando di vivere in sintonia con una dimensione culturale di "debole evitamento dell'incertezza" perché accetto con poco stress di causare un danno importante ad un'altra persona facendo pagare dei costi elevatissimi in termini di difesa della propria faccia negativa. Tale distanza sociale menzionata precedentemente consente l'assenza di identificazione con la persona vittima di tali calunnie. In pratica, l'autore della 
calunnia si trova a proprio agio nell'ambiguità. Questa pratica dell'insinuazione calunniosa viene adoperata spesso nei media per difendere un proprio in-group di riferimento minacciato dalle dichiarazioni offerte dalla persona sottoposta in quel momento a calunnie.

Questo comportamento è possibile perché la società possiede delle opinioni in funzioni delle proprie appartenenze che sentono come inaccettabili le minacce provenienti da altri gruppi percepiti come " outgroup". La persona in sintonia con questo modello può definirsi di appartenere ad una dimensione culturale di tipo "soddisfatto" perché ottiene delle emozioni positive colpendo un'altra persona demolendo il bisogno di preservare la propria faccia negativa della persona colpita dalla calunnia. In sostanza, il calunniatore pratica un atto sadico come per dire "colpisco te e spero un giorno inconsciamente che qualcuno colpisca me". Umberto Eco, in questo articolo, mette in luce come i giornalisti spagnoli avessero una visione troppo vasta di questo concetto della macchina del fango dove per lui sarebbe stato meglio usare la parola "cattiveria" o "malizia" quando ad esempio viene fotografato Varoufakis con la moglie all'interno di un appartamento lussuoso. Per Eco questa fotografia è cattiveria perché serve per mettere in cattiva luce facendo pagare dei costi al bisogno di faccia positiva di Varoufakis riducendo la sua possibilità di ottenere "solidarietà e spirito di associazione" da parte di coloro che sostengono il mondo politico della sinistra. La macchina del fango va a colpire il diritto di protezione della faccia negativa di una persona, ossia la difesa della propria vita privata o intima rendendo pubblico delle allusioni sul modo di vivere la propria vita privata facendoti pagare dei costi enormi alla luce della tua persona come "umana o non umana, normale o strana, sana o folle". In definitiva vengono colpiti le fondamenta della tua identità privata per distruggere la tua influenza nello spazio sociale. Con tale comportamento linguistico vengono meno la massima 
di qualità dicendo "quello che non si può provare", e si dicono cose che non sono pertinenti/rilevanti con la vita professionale di una persona. La stessa massima di maniera viene capovolta perché si sostiene l'oscurità e l'ambiguità dei propri propositi quando si pratica questa "arte" dell'insinuazione calunniosa. Questi atti linguistici odiosi sono possibili perché vige molta distanza sociale nella vita pubblica italiana in termini di differenze socioeconomiche e come appartenenza a differenti in-group. In queste situazioni, la gerarchia sociale significa ineguaglianze esistenziali dato che risulta irrilevante giustificare con $\mathrm{i}$ fatti le proprie affermazioni. Le persone con più potere possono accettare di aderire ad una "dimensione di debole evitamento dell'incertezza" quando compiono tali atti perché il tutto viene vissuto con poca ansia. Inoltre la dimensione di tipo "soddisfatti" si ritrova con la possibilità di vivere emozioni positive con la realizzazione di tali comportamenti.

Umberto Eco, nel primo esempio con Varoufakis, smonta la fotografia conferendo nuovamente apprezzamento per la propria faccia positiva al ex ministro dell'economia greco affermando che non ritroviamo nessun elemento di lusso né nella casa così come nelle pietanze presenti nella fotografia. Umberto Eco menziona che Varoufakis è stato docente in America e pertanto non poteva essere un "povero" e pertanto conferisce faccia positiva offrendo riconoscimento alle competenze professionali del ministro greco. Per Umberto Eco, il caso italiano evidenzia una macchina del fango in cui si vuole alludere, fare delle ipotesi nefaste, creare dei danni concreti per delle condotte private tutte da verificare o accertare come se riguardassero la pubblica opinione, in termini di riduzione di costi per la perdita della loro faccia collettiva di tipo positiva. In questo modo si fa nascere il sospetto come vera tecnica della macchina del fango soprattutto verso gli insospettabili, in modo da collocare tutti sulla stessa barca in Italia, vale a dire gli onesti e disonesti, 
corretti o corrotti. Questa è l'operazione centrale degli ultimi vent'anni in Italia per abortire ogni possibilità di "rinascimento culturale" nel paese, facendo sempre pagare dei costi elevatissimi alla faccia collettiva negativa dei tanti italiani che non vogliono essere "associati" con tali comportamenti. La cultura del sospetto è possibile lì dove non è importante legittimare quello che si afferma, dove prevale un debole evitamento dell'incertezza per gli in-group al potere, a proprio agio nell'ambiguità come terreno privilegiato da coloro che si sentono minacciati da parte di altri piccoli in-group, i quali devono essere schiacciati prima di potere incidere sulla scena mediatica del paese. Aderiscono alla dimensione culturale di essere " soddisfatti" procurandosi delle emozioni positive facendo il male agli altri.

Sostanzialmente, la tecnica è quella di fare apparire in una cattiva luce una persona che non fa niente di male ma allo stesso tempo può sembrare qualcuno che aderisca ad uno stile di vita minoritario nella popolazione benpensante/moralista italiana. Di fondo si deve riportare un elemento vero ma facendo sottintendere qualcosa di negativo in modo che colui che voglia intendere intenda.

Prendiamo l'esempio della fotografia del magistrato seduto nel parco con i calzini turchesi e vestito in modo un po' colorato. Questa foto consente di alludere ad una appartenenza ad una cultura dei figli di fiori da parte del magistrato ad esempio, e questo in un periodo di crisi economica, di restaurazione culturale viene percepito da parte di molti italiani come una minaccia alla loro faccia negativa, ossia alle loro difficoltà per arrivare alla fine del mese per dirla giornalisticamente. Questo meccanismo può tradursi dicendo che tutto quello in apparenza "deviante" è percepito come una minaccia per coloro che non possono mai "deviare" in nulla nella loro vita quotidiana. Per quelli che aderiscono in modo permanente alla dimensione culturale di "forte evitamento dell'incertezza" come unico modo di intendere 
la vita, l'adesione di altri italiani alla dimensione culturale di "debole evitamento dell'incertezza" viene percepita come una minaccia insopportabile per il loro bisogno di faccia positiva, ossia l'assenza di riconoscimento per i loro perenni sacrifici. Tale assenza di riconoscimento per la faccia positiva delle tante persone in difficoltà è la malattia che ha portato alla cultura del sospetto e del risentimento in Italia.

La stessa macchina del fango ha colpito ai vertici del vaticano quando si è affermata la presenza di un tumore al cervello del Papa Francesco. Questa notizia, del tutto infondata, aveva l'obiettivo di ridurre notevolmente il peso delle parole del Papa e pertanto fargli pagare dei costi notevoli per il suo bisogno di faccia positiva, ossia per il riconoscimento della sua figura di guida del mondo cattolico. Per molti gruppi interno al mondo cattolico, la forza carismatica di Papa Francesco è tale da spingerli in ogni modo a ridurre o distruggere la sua potenza oratoria per ridurre i costi subiti da parte di altri gruppi cattolici presenti all'interno della chiesa romana. Lo scopo, in questo caso della macchina del fango, è quello di fare pagare dei costi inaccettabili per ridimensionare tutti i protagonisti della scena sociale o culturale come membri dello stesso in-group. Tale operazione consente di trasformare e rendere tutto il corpo sociale, agli occhi di molti cittadini italiani, come tutti uguali in termini di difetti. In questo modo, i gruppi al potere riescono a ridurre $i$ costi in termini di perdita di faccia positiva imposti da parte di altri gruppi, i quali invece subiscono costi elevatissimi in termini di perdita di faccia positiva con l'assenza di riconoscimento da parte della pubblica opinione per il loro operato. Allo stesso tempo, la loro faccia negativa viene colpita perché non possono preservare il loro diritto di " non associazione" con questo genere di operazione mediatiche.

$\mathrm{Da}$ qui, in molti cittadini onesti ma sempre incerti sulla validità e forza delle loro convinzioni, è nata una cultura dell'indulgenza preventiva in quanto 
il senso comune vuole che tutti al posto dei "potenti" avrebbero agito nello stesso modo. Questo è il risultato più forte ottenuto da parte della macchina del fango e che ha spinto coloro che non aderivano a questo disegno sociale ai margini, al disimpegno o all'espatrio all'estero.

\section{Conclusione}

In questa analisi si è notato come Umberto Eco abbia evidenziato il funzionamento di una macchina del fango in cui si vuole alludere, fare delle ipotesi nefaste, creare dei danni concreti per delle condotte private tutte da verificare come se riguardassero la pubblica opinione. In questo modo si fa nascere il sospetto come vera tecnica della macchina del fango soprattutto verso gli insospettabili, in modo da collocare tutti sulla stessa barca in Italia, vale a dire gli onesti e disonesti, corretti o corrotti. Questo comportamento ha fatto pagare dei costi elevatissimi alla faccia collettiva negativa dei tanti italiani che non vogliono essere "associati" con tali comportamenti. Da qui, in conclusione possiamo dire che molti cittadini onesti hanno aderito ad una cultura dell'indulgenza preventiva in modo da proteggere la propria faccia negativa ma allo stesso volendo rimanere in armonia con la "faccia collettiva" del paese.

\section{Bibliografia}

L'articolo di Umberto Eco La macchina del fango si può ritrovare nel seguente collegamento:

https://espresso.repubblica.it/opinioni/la-bustina-di-

minerva/2015/10/28/news/la-macchina-del-fango-1.23631

BROWN, Penelope. LEVINSON Stephen. Universals in language use: Politeness phenomena, in E. Goody (1978), Questions and politeness. Strategies in social interaction. Cambridge: CUP, 1978.

Social structure, groups and interaction. In: H. Giles e K. R. 
Analisi delle dimensioni culturali e della cortesia linguistica presente nell'articolo La macchina del fango di Umberto Eco

Edoardo Natale

Scherer. Social markers in speech. Cambridge: Cambridge University Press, 1979. Politeness: Some universals in language usage, Cambridge: Cambridge University Press, 1987.

GOFFMAN, Erving. The Presentation of Self in Everyday Life. Penguin Books, 1990.

Doubleday, 1967.

Interactional rituals. Essays on the face-to-face behavior. New York:

. Relations in public. Microstudies of the public order. New York: Harper Torchbooks, 1971. . Modelli di interazione. Bologna: Il Mulino, 1971.

GRICE, Paul. Logic and conversation. In: D. Davidson, G. Harman (eds) The logic of grammar, Encino, California, Dickenson (trad. It. Grice P.(1993), Logica e conversazione, Bologna, il Mulino) 1975.

HOFSTEDE, G. Dimensionalizing cultures: The Hofstede Model in context. Online Readings in Psychology and Culture 2, 2011 Retrieved from http://scholarworks.gvsu.edu/orpc/vol2/iss1/8 